\title{
Quality of Service Workshop
}

\author{
Jan de Meer ${ }^{\mathrm{a}}$ jdm@fokus.berlin.gmd.d400.de \\ Andreas Vogel ${ }^{b} \quad$ andreas@dstc.edu.au \\ ${ }^{a}$ GMD-FOKUS \\ Hardenbergplatz 2 \\ 10623 Berlin, Germany \\ ${ }^{\mathrm{b}} \mathrm{CRC}$ for Distributed Systems Technology \\ DSTC, Level 7, Gehrmann Laboratories \\ University of Queensland, 4072, Australia
}

The 3rd Workshop on Quality of Service (QoS) was held in February 1995 in Brisbane, Australia, in conjunction with the International Conference on Open Distributed Processing. The first workshop took place in May 1994 at the Universite de Montreal, Canada, and the second one during the European RACE Conference on Integrated Services and Networks (IS\&N) in September 1994 in Aachen, Germany. The first two workshops were supported by the European RACE project on QoS TOPIC, and the recent workshop in Brisbane qualified as an IFIP workshop as well. Because of the interest in and the feedback to this series of workshops we are looking forward to another ICODP-workshop on QoS, possibly early in 1996.

The Brisbane workshop addressed the problem of introducing QoS concepts and mechanisms on the different levels of abstraction in a system. The invited talk by GMD-FOKUS Berlin opened the workshop by highlighting the paradigm change which occurs, when moving from message-based systems to continuously transmitting ones. Whereas the former can be checked by a "probe-and-observe" technique, the latter cannot be. There are two major differences. One is the continuous flow of information, which requires continuous observations, and the second is the distribution of applied quality control algorithms. In a client server system, you have admission control procedures applied to the server and deficiency control procedures applied at the client. These QoS control elements cooperate to provide the required QoS level. In order to be confident of the distributed QoS control procedure one has to observe the endto-end behaviour instead of partial behaviours. Message-based systems are separated into protocol entities, which are checked independently because of their point-to-point communication nature.

In a later session, there was a presentation and a discussion of how to classify service qualities for high performance storage systems. An approach to QoS negotiation for multimedia on-demand systems was presented by the Universite de Montreal. HP California looked at how the performance of distributed processing could be improved by the integration of continuously measurement and decision models into the system design and maintainance. Despite 
success in improving system efficiency, many problems are still waiting for a solution. It was recognised that standardised interfaces and the object-based approach of ODP is crucial for the integration of QoS. Thus middleware could aid the decomposition of sophisticated application objects into more measurable ones. The University of Dresden, Germany, presented a tool-based approach which allows the specification, application and monitoring of userdefined QoS parameters for high-speed transport protocols. There is a need for the integration of QoS aspects into the services of distributed platforms. Reconfiguration, an important concept for QoS customisation, addressed by the Norwegian Computing Centre, which participated in an European RACE project on Service Creation. The approach taken is based on the Formal Description Technique SDL and comprises the demands for QoS management of reconfiguration in accordance with invariances of service qualities. A flexible approach to dynamic routing was jointly presented by the Dutch PTT and University of Enschede. Heuristics support the search for an optimal allocation of communication and computation resources when a reconfiguration occurs.

The presentation of papers was followed by a panel which addressed the role of middleware for QoS issues. The panel consisted of Richard Friedrich (HP California), Jerry Rolia (Carleton University Ottawa), Jan de Meer (GMD-FOKUS Berlin), Jacob Slonim (Centre of Advanced Studies IBM Toronto) and Andreas Vogel (DSTC). It was said that cost and security must play an important role in the evaluation of service qualities. The acceptance of the needs of specialised groups of users is not yet sufficiently studied. Despite the technical qualities, user groups expect sophistication of security and privacy policies at reasonable cost. The more technical memebers of the audience thought that economic factors such as cost lead to a discussion outside the ODP framework. It was recognised that economic aspects are of great importance for the discussion on QoS. However, a relation was tackled to trading based on service qualities. Formal description techniques did not play an important role during discussion.

We would like to thank all panelists and others who contributed to the success to this ICODP workshop. We hope that the issues on QoS addressed in this workshop become more aligned to the user's and supplier's needs. 